\title{
SMC protein RecN drives translocation and remodelling of RecA filament for homology search
}

\author{
Afroze Chimthanawala, Jyotsana Parmar, Sujan Kumar, \\ Krishnan S. Iyer, Madan Rao*, Anjana Badrinarayanan* \\ National Centre for Biological Sciences (TIFR), Bengaluru 560065, India
}

${ }^{*}$ To whom correspondence should be addressed;
E-mail: madan@ ncbs.res.in, anjana@ncbs.res.in

While the molecular repertoire of the homologous recombination pathway is well studied, the search mechanism that enables recombination between distant homologous regions is poorly understood. Here, we follow the dynamics of the recombinase RecA, an essential component of homology search, after induction of a single double-strand break on the Caulobacter chromosome. We find that the RecA-nucleoprotein filament translocates in a directional manner in the cell, undergoing several pole-to-pole traversals, until homology search is complete. Simultaneously, the filament undergoes dynamic remodelling; both translocation and dynamic remodelling are contingent on the action of the SMC protein RecN via its ATPase cycle. We provide a stochastic description of RecN regulated changes in filament length during translocation via modulation of RecA assembly-disassembly. Together, the observed RecN driven RecA dynamics points to a novel optimal search strategy.

Keywords: homology search, homologous recombination, double-strand break, bacteria, RecA, 
Structural Maintenance of Chromosome proteins, RecN, live-cell imaging, stochastic dynamics, assembly-disassembly

\section{Introduction}

DNA double-strand breaks (DSBs), if left unrepaired (or incorrectly repaired) can lead to loss of genetic information, chromosomal rearrangements, mutagenesis and even cell death. Homologous recombination, a process that usually ensures error-free repair of DSBs has emerged as an evolutionarily conserved pathway from bacteria to eukaryotic cells $(1,2)$. Recent work has shown that recombination-based repair is not only local, such as during post-replicative cohesion, but that even distant homologous regions can participate in such repair (2-6). This is particularly relevant for bacteria, where an extensive post-cohesion period is absent. Since DSBs can occur independent of the replicative phase of the cell cycle and that even a single unrepaired DSB can be potentially lethal, it is vital that there be regulatory mechanisms to sense and initiate, translocate and search and finally identify distant homology regions for repair. These strategies for long distance homology search and repair must potentially depend on the spatial organisation of chromosomes - its extent, geometry and dimensionality (7) - and must involve energy transduction.

In bacteria, for instance, an extensive homology search programme is effected by the highly conserved recombinase, $\operatorname{Rec} A(8-10)$. In the first step of the recombination pathway, DSB-ends are processed to reveal ssDNA overhangs, on which RecA can assemble into a filamentous structure $(4,11,12)$ which in turn initiates homology search. In vitro studies have shown that RecA molecules in the nucleoprotein filament possess ATPase activity that can influence (a) formation of a continuous filament during RecA loading on ssDNA (13), (b) growth and shrinkage of the filament (14-16), (c) release of the filament from heterologous pairing $(9,17)$, and (d) RecA turnover during strand invasion (16-18). In vivo imaging of RecA in E. coli has 
revealed the presence of large assemblies of RecA, described as RecA filaments (or 'bundles'), that can even span the length of the cell $(5,6)$.

In addition, several accessory proteins are reported to modulate the association and turnover of RecA with ssDNA $(19,20)$. Of these, RecN, a highly conserved repair-associated protein, has been shown to interact with RecA and is essential for DSB repair (20-23). RecN belongs to the Structural Maintenance of Chromosome (SMC) family of proteins, that play central roles in chromosome dynamics (24). While their function in chromosome organization and segregation in bacteria has been well-characterized, their role in DNA repair pathways has not been clearly established. Previous studies have suggested a role for $\mathrm{RecN}$ in homologous recombination, either via its influence on kinetics of strand-invasion by the RecA filament or by facilitating global chromosomal cohesion between replicated sisters $(20,22,25,26)$. Indeed the potential relevance of RecN in recombination-based repair is underscored by its extensive conservation with other core recombination-associated proteins such as RuvABC and RecA across bacterial genomes, as well as across domains of life (27).

In conjunction with other molecular regulators, homology search is an efficient and robust process, typically completed within a single generation time of bacterial growth $(3,4,6)$. How RecA orchestrates this search, the dynamics of the nucleoprotein filament during this process, and the role for RecA regulators in facilitating or modulating homology search, remain largely unknown. To elucidate the mechanism of homology search in vivo, we use quantitative live cell imaging to track RecA during DSB repair in the bacterium Caulobacter crescentus, together with a theoretical description of RecA dynamics during search.

Here we present evidence for a search mechanism involving a RecA nucleoprotein filament that translocates in a directional manner from the break site located at one cell pole, to the opposite cell pole. Simultaneously, the RecA filament undergoes dynamic changes to its structure, while traversing back-and-forth across the length of the cell before finding its homologous part- 
ner and initiating homology pairing. Both, large scale movement and dynamic remodelling of RecA are contingent on its association with RecN. We present a stochastic description, where RecN influences dynamic changes in RecA filament lengths via regulation of rates of RecA subunit assembly-disassembly. Such dynamical remodelling and directional movement of the RecA nucleoprotein filament points to a robust mechanism of iterative search, thus enabling recombination between distant sites of homology.

\section{Results}

\section{In vivo imaging of RecA reveals key steps of homologous recombination}

To follow RecA-dependent search dynamics in vivo, we use the I-SceI system developed in Caulobacter crescentus $(4,12)$ that allows us to visualize DSB repair between distant homologous regions (Fig. S1A). Briefly, we insert a unique restriction site, I-SceI, on the bacterial chromosome near the polarly localized origin of replication. We induce a DSB by regulated expression of the endonuclease, I-SceI, that cleaves the site to generate a break. We further marked a region of the chromosome near the break-site using fluorescently-tagged MipZ that associates with the origin region. DSB induction results in loss of localization of the MipZ marker close to the break (due to processing of DNA by the helicase-nuclease complex AddAB $(4,12))$ serving as a reliable proxy to identify cells where only a single copy of the chromosome is cleaved (with only one MipZ marker now being visible). Restoration of the second marker is indicative of repair.

Using this extensively characterized system, we follow the process of homology search in two distinct cell types, both blocked for new rounds of replication initiation: pre-divisional cells with two completely replicated and segregated chromosomes (to follow homology search and subsequent recombination-mediated repair of distant homologous regions) and non-replicating swarmer cells with a single chromosome (to follow homology search, without the presence of 
the homologous repair template) $(4,12)$.

In both cell types, we monitor the dynamics of RecA using live cell imaging of RecAYFP, expressed ectopically in a wild type RecA background (4, 5, 28). This strain, previously described in (4), is comparable to the wild type background in terms of function in generic DNA damage repair, recombination-mediated DSB repair, as well as SOS response induction (Fig. S1B-D). We find that Caulobacter RecA-YFP forms discrete structures (foci or elongated filaments) only in the presence of DNA damage. In the absence of damage, fluorescence is diffuse throughout the cell (Fig. S4F). We observe three distinct stages following a DSB near the cell pole - (i) nucleation and growth of RecA filament near the pole where MipZ localization, proximal to the origin $(4,12)$, is lost (indicating occurrence of a DSB), (ii) homology search, where the RecA filament remodels and moves directionally across the length of the cell (expanded in the next sections), and (iii) repair, associated with RecA dissociation followed by the reappearance of two distinct MipZ markers (Fig. 1A, B).

\section{Growth and directional movement of RecA filament during homology search}

We now characterize the dynamics of RecA during the homology search phase. In contrast to the static structures previously reported in $\operatorname{E}$. coli $(5,6)$, we find that once RecA nucleates at the DSB site near the pole, it rapidly grows into an elongated filament, following which it dynamically moves across the length of the cell (Fig. 1B,C, S2A, S2D; Supplementary Video 1). We follow the time series of the centroid position $\mathrm{x}_{c}(t)$ of the RecA filament along the long-axis of the cell, relative to the cell length $L$ (Supplementary Results for details of quantitative analysis). From its initial position $\mathrm{x}_{c}(0)$ close to the nucleation site, we find that the centroid progresses in a stochastic but biased manner, towards the opposite cell pole (Fig. 1D,E). Having reached the opposite cell pole, the RecA filament starts translocating in the reverse direction (Fig. 1C, S2A). Defining a pole-to-pole translocation as a traversal, we find that the mean traversal time of the 
RecA filament is $6 \pm 2 \mathrm{~min}$, and typically undergoes multiple traversals (mean $=4$ ) before homology pairing (Fig. 1D).

The centroid position is uniformly distributed across the cell length (Fig. 1E, inset). This indicates that there are no hot-spots within the cell that specifically regulate RecA filament dynamics. The distribution of the centroid steps $\Delta \mathrm{x}_{c}$, the algebraic difference between the centroid positions at two consecutive time points, exhibits a bias towards positive values, indicating a systematic movement of the RecA filament in one direction. The nonzero weight of this distribution at $\Delta \mathrm{x}_{c}=0$ is an indication that the RecA filament often stalls (Fig. 1E).

Significantly, we observe that when the DNA break is made midcell $(+780 \mathrm{~kb}$ from the origin) (4), the RecA filament nucleates near midcell, and moves towards either one of the cell poles (Fig. S2B). Similarly, in non-replicating swarmer cells with a single copy of the chromosome, the RecA filament seems to exhibit interminable pole-to-pole traversals (Fig.2F, S2C; Supplementary Video 2). These observations imply that the homology search dynamics proceeds independent of the homologous repair template. Interestingly, although there are two break ends upon DSB induction, we rarely observed two distinct RecA filaments. Thus, RecA appears to form a single filamentous structure spanning both break ends, which translocates across the cell during homology search.

\section{RecN is essential for movement of the RecA filament in vivo}

We now inquire into the molecular basis of the dynamics of the RecA filament during homology search and for holding break ends together during filament translocation. An essential and highly conserved member of the bacterial recombination pathway is the SMC protein, RecN $(21,23)$. However, it still remains unclear as how RecN influences RecA-mediated homologous recombination in vivo. Given the universal role of SMC proteins in organizing DNA (for example, via DNA bridging or DNA loop extrusion (24)), we asked whether RecN contributed 
to RecA dynamics and/ or organization of break ends specifically during homology search.

Consistent with reports in other bacterial systems $(21,23)$, we find that $\mathrm{RecN}$ is essential for successful DSB repair in Caulobacter. In absence of recN we record no detectable repair events, together with an increased sensitivity to DSB-inducing agents (Fig. S3E-F, S4G). We use the bacterial-two-hybrid system to show that Caulobacter RecA and RecN physically interact with each other (Fig. S3D). We also find that the recruitment of RecN to damage sites is RecA dependent (and not only on SOS-dependent expression) (Fig. S3A,B) . Furthermore, RecA and RecN are colocalized at a DSB site, with no detectable instances of RecN localizing away from the RecA structure. However, we do observe examples of RecN localizing towards one end of an elongated RecA filament (Fig. S3C).

Given these observations, we investigate how RecN impacts RecA dynamics and homology search. For this, we first delete recN and follow the dynamics of RecA during the search process via live-cell imaging. As in case of wild type, we still observe a single RecA localization in $\Delta r e c N$ cells, suggesting that RecN does not contribute to holding break ends together. Instead, we find that upon $\operatorname{recN}$ deletion, the RecA filament ceases to translocate in pre-divisional cells (Fig. 2A-D; Supplementary Video 3). Unlike wild type, the RecA filament remains rooted to the pole where the break occurred (Fig. 2B, C, D (inset)) and does not undergo pole-to-pole traversal over the entire course of imaging, up to 100 min (Fig. 2C). Concomitantly, no RecA dissociation is observed, consistent with the idea that recombination between distant homologous regions involves RecN-driven RecA movement.

We find a similar effect on the RecA filament translocation in the absence of $r e c N$, in nonreplicating swarmer cells with only a single copy of the chromosome, in support of our assertion that RecN-driven RecA movement for homology search occurs independent of a homologous template for repair (Fig. 2E, F; Supplementary Video 4). Finally, these perturbations to RecA dynamics are observed only in cells lacking the repair-specific SMC protein $(\operatorname{RecN})$ and not 
the SMC protein (Smc) involved in chromosome organization and segregation in non-damage conditions (24) (Fig. S3F, G).

To study which aspects of RecA activity is perturbed in the absence of recN, we assess the effects of $\operatorname{rec} N$ deletion on kinetic rates at different stages of the RecA dynamics. In the nucleation stage, we measure the time taken from MipZ disappearance to the appearance of a RecA nucleation event at a DSB site, and find it comparable to wild type cells (Fig. S4A). In the growth stage following nucleation (and prior to search), we find that the initial rates of RecA loading are unaltered in recN deleted cells (Fig. S4D, E). In line with this, we observe that the frequency of RecA nucleation events across $\Delta r e c N$ cells is comparable to wild type, even under generic mitomycin-C (MMC) damage (Fig. S4F).

Finally, we examine whether the absence of RecA filament movement in $r e c N$ deleted cells is a consequence of a RecA filament instability. Previous studies have shown that cells lacking recA or with mutants of RecA that are unable to stably associate with a DSB, undergo 'recless' degradation, resulting in excessive loss of DNA around a break site $(8,29)$. Hence we measure DNA degradation in cells with a single DSB $(+780 \mathrm{~kb}$ from the origin in non-replicating swarmer cells) in wild type, $\triangle r e c A$ and $\Delta r e c N$ backgrounds, via a deep-sequencing assay (12, 30). Our results show that cells lacking recN have degradation profiles that overlap with that of wild type (Fig. S4B, C), while cells lacking recA show extensive loss of DNA around the break site. In addition, $\Delta r e c N$ cells do not have perturbed SOS induction (Fig. S4H). These observations suggest that RecA filament association with a DSB is not affected in the absence of recN, although its movement during homology search is stalled. 


\section{RecN-mediated RecA translocation is accompanied by dynamic remod- elling of the filament during homology search}

Together with the observation that $\mathrm{RecN}$ does not affect RecA filament formation at a break site, we find that the average filament lengths are comparable in both wild type and $\Delta r e c N$ cells. The length of the RecA filament is typically a small fraction of the cell length and does not extend over the entire cell. In wild type, filament mean fractional length is $\langle l\rangle=0.2 \pm 0.1$ (Fig. 3E), that corresponds to $1.0 \pm 0.5 \mu \mathrm{m}$ for cell lengths that range between $5-6 \mu \mathrm{m}$. In case of $\Delta \operatorname{rec} N$ cells, mean fractional length is $\langle l\rangle=0.2 \pm 0.06$, that corresponds to $1.2 \pm 0.5 \mu \mathrm{m}$ for cell lengths that range between $7-9 \mu \mathrm{m}$ (Supplementary Results). However, although the mean filament lengths are similar, we note that the distribution of $\Delta l$, the algebraic difference between the filament lengths at two consecutive time points shows a large range in case of wild type cells (Fig. 3A). This suggests that the filament is dynamically remodelling during translocation, both of which appear to be compromised in the absence of $\operatorname{recN}$ (Fig. 2D, 3A).

Indeed, in wild type cells, we find that directional movement of RecA during homology search is accompanied by dynamic changes in the RecA filament over time. We observe various types of RecA structures in the cell during a traversal, ranging from a foci to extended filamentous structures (Fig. 3B). Importantly, we find no correlation between the length of the RecA filament and its centroid position in the cell (Fig. 3C). Thus, RecN-assisted movement of the RecA filament is accompanied by a stochastic remodelling of its length.

How does RecN regulate RecA filament dynamics? The stochastic remodelling of RecA filament length during homology search suggests to us that RecN action must influence assemblydisassembly rates of RecA molecules on the DNA strand (akin to treadmilling) during translocation. Thus, to understand the contribution of assembly-disassembly rates on the observed RecA filament lengths during translocation, we resort to a quantitative stochastic description of the mechanochemistry of RecN-assisted RecA filament dynamics (Supplementary Results). 
In general, the stochastic kinetic model is parameterized by four kinetic rates acting at the ends of the RecA filament (Fig. 3D): a binding rate $k^{+}$at the left end, a binding rate $k^{++}$at the right, an unbinding rate $k^{-}$at the right, and a $\mathrm{RecN}$-dependent unbinding rate $k^{--}$at the left (noting that the polarity of the filament (and RecA ATPase states) that dictates directionality and assembly-disassembly of RecA molecules (9, 14, 19, 31-33)).

We first note that in vivo, the levels of RecA ((5) and Fig. S4E) and ATP (34) are likely not limiting. With this in mind, if the assembly-disassembly rates are taken to have fixed values, then they cannot be made to balance each other robustly at a particular length and will not give rise to a finite mean filament length at steady state as is observed for wild type (Fig. 3E). One needs a negative feedback between the assembly-disassembly rates and the filament length (Fig. 3D, Supplementary Results) in order to provide a homeostatic balance leading to a finite mean filament length with small fluctuations about it (35). We solve this stochastic kinetic model with length dependent rates analytically using a Langevin dynamics with multiplicative noise (Supplementary Results). We see that the net bias of the filament to move to the right (Fig. 1E), is contingent on two key features (Supplementary Results) - (i) the kinetics rates must violate detailed balance and must thus be driven by energy consumption and (ii) there is a net breaking of left-right symmetry in the binding-unbinding rates, i.e. the net binding at the right end of the filament is larger than the net unbinding at the left end (in the pure assemblydisassembly limit, this is akin to treadmilling (36)).

We obtain closed form expressions for the steady state distribution of the filament length $P(l)$ (Supplementary Results), which we compare with the observed distribution for the wild type cells (Fig. 3E). With the disassembly rate $k^{--}$varying with length as in Fig. 3D, we find that the analytically derived steady state $P(l)$ is a perfect match to the observed distribution (Fig. 3E). In addition, we predict a linear correlation between the mean centroid velocity and mean filament length at steady state, which appears consistent with the wild type data (Fig. 3E 
(inset)). Thus, our stochastic theory provides a framework for how RecN-mediated regulation of assembly-disassembly rates of RecA subunits within a filament can result in the filament length dynamics observed during homology search.

\section{RecN ATPase cycle regulates RecA filament remodelling}

While the molecular mechanism of filament remodelling and its impact on translocation warrants future investigations (see below), the following additional observation underscores a central role for RecN in modulating RecA filament lengths: A fundamental characteristic of SMC family proteins is their ATPase cycle, that enables them to influence the organization of the substrates they are bound to as well as translocate across large distances within the cell (37-39). We hence asked how the RecN ATPase cycle regulates RecA filament dynamics, thus facilitating recombination between distant homologous regions. For this, we generate two mutants of RecN in the conserved WalkerB motif that are likely to result in specific perturbation to either ATP binding or ATP hydrolysis properties of $\operatorname{RecN}(37,40): r e c N^{D 472 A}$ (ATP-binding impaired) and $\operatorname{recN}^{E 473 Q}$ (ATP-hydrolysis impaired). We find that both mutants are still able to interact with RecA, but are compromised in DNA damage repair, as seen in case of recN deletion (Fig. 4C,D). We next follow the dynamics of RecA during homology search after induction of a DSB in cells carrying either the $\operatorname{rec} N^{D 472 A}$ or $r e c N^{E 473 Q}$ mutations. We find that these mutants also result in static RecA filaments that are unable to translocate across the cell for homology search (Fig. 4A, B, E-G; Supplementary Video 5-6). While translocation of RecA is similarly impaired in both mutants, we notice that RecA filament lengths are differently affected. In case of $r e c N^{D 472 A}$, RecA filament lengths are comparable to wild type, while they are significantly shorter in the RecN ATP hydrolysis mutant (Fig. 4H).

Taken together, our observations position RecN early in the recombination pathway, specifically in enabling RecA filament translocation during homology search, with influence on RecA 
dynamics even in the absence of a homologous repair template. The differential impact of RecN ATPase mutants on RecA filament lengths (both resulting in abrogation of translocation), leads us to suggest that RecN, via its ATPase cycle, modulates RecA nucleoprotein filament remodelling and drives directional translocation of the filament during homology search.

\section{Discussion}

Using a combination of quantitative live cell imaging and theoretical modelling, we have uncovered a new mechanism for RecA action in long distance homology search during recombination, a fundamental process for the maintenance of life. Our first result is that following a site-specific DSB, RecA nucleates at the DSB site and grows into a nucleoprotein filament which moves in a stochastic but directional manner along the length of the cell, in tandem with RecN. The second main result is that the filament translocation is accompanied by dynamic remodelling of the RecA filament. The dynamic remodelling is a consequence of RecN influenced assembly-disassembly that breaks left-right symmetry (akin to treadmilling) - indeed, a stochastic description of this process agrees remarkably well with quantitative analysis of live cell imaging. The RecA filament makes multiple traversals across the length of the cell before the homologous pair is found, followed by repair. That RecN, via its ATPase cycle, is crucially involved in the search, its termination and subsequent repair is the third main result of the paper.

Following our present study, an immediate goal is to deduce the molecular basis of the feedback regulation that maintains a fixed mean filament length at steady state. Similarly, we will need to unravel the molecular basis of the RecN-regulated dynamics of the RecA nucleoprotein filament. This would require a quantitative characterization of the complete RecA-RecN ATPase cycle and its interaction with the mechanochemistry of the DNA substrate.

Based on our current observations, we consider two possible scenarios: (A) RecN-mediated regulation of assembly-disassembly rates of RecA molecules in the filament. (B) Motor-driven 
movement of the RecA nucleoprotein filament and/ or the underlying ssDNA substrate. In line with the possible influence of RecN on mobility of the underlying ssDNA substrate, we find that, in the absence of $\operatorname{recN}$, Single-Strand Binding protein (SSB) localization is also restricted to the pole where the break occurs (Fig. S5A). However, pure motor-assisted movement would not be able to explain the stochastic remodelling the filament length, while pure assemblydisassembly, without the dynamics of the DNA, would need to have available a cell-spanning ssDNA segment, which does not appear to be the case in our conditions (Fig. S5A).

Thus, we suggest that the RecN-assisted dynamics of the RecA filament could be a consequence of both motor-assisted movement (such as DNA extrusion) and regulation of assemblydisassembly rates of RecA on DNA (via treadmilling). Fig. S5B is our proposed model of the same. Here, RecN action on the DNA could be a consequence of its reported bridging activity of ssDNA (generated around the break-site) with dsDNA) (26), thus driving directional movement of RecA, along with its underlying DNA substrate. 'Zipping' refers to RecN action ahead of the translocating RecA filament, while 'extrusion' refers to $\mathrm{RecN}$ action behind the filament $(41,42)$.

Why does the RecA filament need to undergo dynamic remodelling in addition to movement during homology search? Studies have shown that RecA ATPase rates are stimulated 4-fold upon strand invasion, potentially allowing the RecA filament to release from heterologous pairing during microhomology sampling as well (17). Separately, RecN has been found to (a) stimulate RecA strand invasion activity (20) and (b) bridge ssDNA and dsDNA (26). Taken together, it is possible that the RecN-dependent RecA remodelling is a reflection of such microhomology sampling activity prior to homology pairing.

The stochasticity of the RecN-driven RecA dynamics and the multiple traversals before identification of the homologous repair template, suggests a novel search mechanism that allows for a regulated genome-wide sampling for homology. Our observations reveal that in addition 
to the initial waiting time between nucleation to the start of traversals, the translocating RecA filament makes pauses at random intervals. We speculate that RecA molecules might engage in microhomology sampling both initially in the vicinity of the damage site (especially in the post-replicative cohesion period) and during these pause intervals during translocation $(17,43)$.

Based primarily on in vitro observations, earlier models have suggested that RecA engages in 1D sliding movement and intersegment hopping, while regions within the RecA filament engage in microhomology sampling during search $(9,43,44)$. The requirement for $\mathrm{RecN}$ in driving homology search in vivo underscores an additional layer of critical regulation that is central to RecA function. RecN-mediated multiple pole-to-pole traversals by RecA builds in a kind of reset at a fixed spatial location, a strategy that can lead to optimal search (45).

It is possible that there is some diversity in RecA-dependent long-range homology search dynamics across bacterial systems, to contend with the varying cell shapes, chromosome replication dynamics (multi-fork vs replication only once per cell cycle), and chromosome organization (3-6). However, given that RecA and RecN are among the most conserved proteins of the recombination pathway in bacterial genomes and its counterparts are present across domains of life, we believe that the dynamics we describe here is active in other systems as well.

\section{Acknowledgements}

$\mathrm{AC}$ and $\mathrm{AB}$ thank Julia Hitschfel for assistance with bacterial-two-hybrid experiments as well as Meghna Iyer and Varshit Dusad for assistance with developing analysis tools. AC and AB are grateful to Rodrigo Reyes-Lamothe, Christian Lesterlin, David Sherratt, M Srinivasan and AB lab members for feedback and discussion. AB acknowledges support from DST-SERB CRG 2019/003321, HFSP-CDA award (00051/2017) and intramural funding from NCBS-TIFR. JP, SK, KI and MR thank the Simons Foundation for a grant. MR thanks DST-SERB, India for a JC Bose Fellowship. 


\section{Materials and Methods}

\section{Bacterial strains and growth conditions}

Strains, plasmids and oligos used in this study are described in Table S1-S3, respectively. Chromosomal modifications such as integration of fluorescent markers or deletion of genes were performed using the two-step recombination method (46) or with vectors described in (47). Transductions were carried out using $\Phi \mathrm{Cr} 30$ (48). Unless otherwise stated, Caulobacter cultures were grown at $30^{\circ} \mathrm{C}$ in peptone yeast extract and supplemented with antibiotics at appropriate concentrations. When growing strains with dnaA under an IPTG-inducible promoter, liquid media was supplemented with $0.5 \mathrm{mM}$ IPTG and solid media with $1 \mathrm{mM}$ IPTG. For synchronization experiments, Caulobacter cultures were grown till mid-log phase and synchronization protocols were followed as described in (4). Protocols used for isolating non-replicating pre-divisional cells and non-replicating swarmer cells $(4,12)$ are briefly described here. For isolation of non-replicating swarmer cells, cultures were grown till mid-log phase in media supplemented with IPTG. Cells were then depleted for DnaA by washing off the inducer and were grown in the absence of IPTG for one generation. This was followed by synchronization and isolation of swarmer cells. These cells were re-suspended in media without IPTG and used for DSB induction experiments. For isolation of non-replicating pre-divisional cells, cultures were grown till mid-log phase in media supplemented with IPTG. Cells were then synchronized and swarmer cells isolated. Swarmers were then released into media lacking IPTG. Cells were then depleted for DnaA by washing off the inducer and grown in the absence of IPTG for one generation. Cell division was blocked via addition of $35 \mu \mathrm{g} / \mathrm{ml}$ cephalexin and these non-replicating pre-divisional cells were further used for DSB induction experiments. In all cases, recA-YFP was induced using $0.03 \%$ xylose 90 min prior to imaging and subsequently maintained in the agarose pad as well during the entire course of imaging. For imaging SSB-YFP after induction 
of a break in non-replicating swarmer cells, $s s b$-YFP was induced using $0.03 \%$ xylose. DSBs were induced with $2 \mu \mathrm{M}$ Vanillate. For this, vanillate was added to the growth culture $15 \mathrm{~min}$ prior to imaging and was subsequently maintained on the agarose pad as well.

\section{Fluorescence Microscopy}

Time course imaging was performed on $1 \%$ agarose pads (Invitrogen ultrapure). For time-lapse imaging, cells were grown on 1.5\% GTG agarose pads (low melting) prepared in peptone yeast extract and supplemented with vanillate, xylose or cephalexin at appropriate concentrations and imaged using glass-bottom petri dishes. Imaging was carried out using a wide-field epifluorescence microscope (Eclipse Ti-2E, Nikon) with a 63X oil immersion objective (plan apochromat objective with NA 1.41), illumination from pE4000 light source (CoolLED), Hamamatsu Orca Flash 4.0 camera and a motorized XY stage. During time-lapse imaging, focus was maintained using an infrared-based Perfect Focusing System (Nikon). Image acquisitions were done using NIS-elements software (version 5.1) and images were acquired every 30s, 1 min or 5 min (as indicated in the main text and respective figure legends). For most experiments with RecA, exposure time used for excitation at $490 \mathrm{~nm}$ was $400 \mathrm{~ms}$ and for $550 \mathrm{~nm}$ was $500 \mathrm{~ms}$. For imaging MipZ, exposure time used for excitation at $550 \mathrm{~nm}$ was $100 \mathrm{~ms}$. For imaging YFP expressed from the sidA promoter, exposure time used for excitation at $490 \mathrm{~nm}$ was $500 \mathrm{~ms}$. In all images, scale bars $=2 \mu \mathrm{m}$. Procedures for image analysis and for extracting key features of RecA localization are described in Supplementary Results.

\section{DNA degradation assay}

Implemented as described in (12). Briefly, non-replicating swarmer cells with DSB site at $+780 \mathrm{~kb}$ were isolated and released in a media with $0.5 \mathrm{mM}$ Vanillate to induce DSBs. Samples were collected at $0 \mathrm{~h}$ (control) and $1 \mathrm{~h}$ after DSB induction. Genomic DNA was isolated 
using DNAeasy blood and tissue kit (Qiagen). Whole genome Illumina sequencing was carried out on the isolated DNA (NGS facility, NCBS, India). Data were further analysed using the following protocol. First, indexing with the reference genome (4.01 Mbp) (NCBI reference sequence:NC-011916.1) was done using BWA (49). Reads with raw read quality 20 were aligned using 'BWA aln -q'. SAMTOOLS (version 0.1.19-96b5f2294a) (49) was used to filter out the multiply-mapped reads. Finally, with BEDTOOLS (50) the read count per bin was calculated using the .bed files containing bin positions. Caulobacter genome data obtained after Illumina sequencing, Hiseq 2500 illumina short reads (50 bp), was divided into $1 \mathrm{~kb}$ bins. Read counts per bin were normalized to the total reads acquired for that sample. The ratio of normalized reads after DSB $(1 \mathrm{~h})$ to normalized reads before DSB $(0 \mathrm{~h})$ was plotted to visualize the read enrichment profile obtained across the genome after induction of a DSB. The graph generated was processed further with the Lowess smoothing function in MatLab.

\section{Bacterial two-hybrid assay}

Implemented as described in (12). Briefly, to investigate physical interaction between a pair of proteins, their respective genes were fused to $3^{\prime}$ end of T25 or T18 fragments in pKT or pUT vectors. These vectors were co-transformed into E. coli BTH101. Co-transformed cells were grown to saturation in M63 media with maltose and IPTG and $5 \mu$ l of this culture was spotted on MacConkey agar plates (40 g/L) with maltose, IPTG and appropriate antibiotics. Plates were incubated at $30^{\circ} \mathrm{C}$ for 2-3 days. 


\section{References and Notes}

1. J. Renkawitz, C. A. Lademann, S. Jentsch, Mechanisms and principles of homology search during recombination. Nature Reviews Molecular Cell Biology. 15, 369-383 (2014).

2. J. E. Haber, DNA Repair: The Search for Homology. BioEssays. 40, 1700229 (2018).

3. V. Amarh, M. A. White, D. R. F. Leach, Dynamics of RecA-mediated repair of replicationdependent DNA breaks. Journal of Cell Biology. 217, 2299-2307 (2018).

4. A. Badrinarayanan, T. B. K. Le, M. T. Laub, Rapid pairing and resegregation of distant homologous loci enables double-strand break repair in bacteria. Journal of Cell Biology. 210, 385-400 (2015).

5. C. Lesterlin, G. Ball, L. Schermelleh, D. J. Sherratt, RecA bundles mediate homology pairing between distant sisters during DNA break repair. Nature. 506, 249-253 (2014).

6. J. Wiktor, A. H. Gynna, P. Leroy, J. Larsson, G. Coceano, I. Testa, J. Elf, Live cell imaging reveals that RecA finds homologous DNA by reduced dimensionality search (bioRxiv) doi:10.1101/2020.02.13.946996 (2020).

7. O. Bénichou, C. Loverdo, M. Moreau, R. Voituriez, Intermittent search strategies. Rev. Mod. Phys. 83, 81-129 (2011).

8. N. S. Willetts, A. J. Clark, Characteristics of Some Multiply Recombination-Deficient Strains of Escherichia coli. J Bacteriol. 100, 231-239 (1969).

9. J. C. Bell, S. C. Kowalczykowski, RecA: Regulation and Mechanism of a Molecular Search Engine. Trends in Biochemical Sciences. 41, 491-507 (2016). 
10. M. M. Cox, Regulation of Bacterial RecA Protein Function. Critical Reviews in Biochemistry and Molecular Biology. 42, 41-63 (2007).

11. G. R. Smith, How RecBCD Enzyme and Chi Promote DNA Break Repair and Recombination: a Molecular Biologist's View. Microbiology and Molecular Biology Reviews. 76, 217-228 (2012).

12. A. Badrinarayanan, T. B. K. Le, J.-H. Spille, I. I. Cisse, M. T. Laub, Global analysis of double-strand break processing reveals in vivo properties of the helicase-nuclease complex AddAB. PLoS Genet. 13, e1006783 (2017).

13. S. H. Kim, T. Ahn, T. J. Cui, S. Chauhan, J. Sung, C. Joo, D. Kim, RecA filament maintains structural integrity using ATP-driven internal dynamics. Sci. Adv. 3, e1700676 (2017).

14. S. H. Kim, K. Ragunathan, J. Park, C. Joo, D. Kim, T. Ha, Cooperative Conformational Transitions Keep RecA Filament Active During ATPase Cycle. J. Am. Chem. Soc. 136, 14796-14800 (2014).

15. M. T. J. van Loenhout, T. van der Heijden, R. Kanaar, C. Wyman, C. Dekker, Dynamics of RecA filaments on single-stranded DNA. Nucleic Acids Research. 37, 4089-4099 (2009).

16. J. M. Cox, O. V. Tsodikov, M. M. Cox, Organized Unidirectional Waves of ATP Hydrolysis within a RecA Filament. PLoS Biol. 3, e52 (2005).

17. J. Y. Lee, Z. Qi, E. C. Greene, ATP hydrolysis Promotes Duplex DNA Release by the RecA Presynaptic Complex. Journal of Biological Chemistry. 291, 22218-22230 (2016). 
18. J. E. Lindsley, M. M. Cox, Assembly and disassembly of RecA protein filaments occur at opposite filament ends. Relationship to DNA strand exchange. Journal of Biological Chemistry. 265, 9043-9054 (1990).

19. J. C. Bell, J. L. Plank, C. C. Dombrowski, S. C. Kowalczykowski, Direct imaging of RecA nucleation and growth on single molecules of SSB-coated ssDNA. Nature. 491, 274-278 (2012).

20. L. A. Uranga, E. D. Reyes, P. L. Patidar, L. N. Redman, S. L. Lusetti, The cohesin-like RecN protein stimulates RecA-mediated recombinational repair of DNA double-strand breaks. Nat Commun. 8, 15282 (2017).

21. S. M. Pieksley, P. V. Attfield, R. G. Lloyd, Repair of DNA double-strand breaks in Escherichia coil K12 requires a functional recN product. Mol Gen Genet. 195, 267-274 (1984).

22. E. Vickridge, C. Planchenault, C. Cockram, I. G. Junceda, O. Espéli, Management of E. coli sister chromatid cohesion in response to genotoxic stress. Nat Commun. 8, 14618 (2017).

23. A. N. Klimova, S. J. Sandler, An Epistasis Analysis of recA and recN in Escherichia coli K-12. Genetics. 216, 381-393 (2020).

24. S. Nolivos, D. Sherratt, The bacterial chromosome: architecture and action of bacterial SMC and SMC-like complexes. FEMS Microbiol Rev. 38, 380-392 (2014).

25. E. D. Reyes, P. L. Patidar, L. A. Uranga, A. S. Bortoletto, S. L. Lusetti, RecN Is a Cohesinlike Protein That Stimulates Intermolecular DNA Interactions in Vitro. Journal of Biological Chemistry. 285, 16521-16529 (2010). 
26. K. Keyamura, T. Hishida, Topological DNA-binding of structural maintenance of chromosomeslike RecN promotes DNA double-strand break repair in Escherichia coli. Commun Biol. 2, 413 (2019).

27. E. P. C. Rocha, E. Cornet, B. Michel, Comparative and Evolutionary Analysis of the Bacterial Homologous Recombination Systems. PLoS Genet. 1, e15 (2005).

28. N. Renzette, N. Gumlaw, J. T. Nordman, M. Krieger, S.-P. Yeh, E. Long, R. Centore, R. Boonsombat, S. J. Sandler, Localization of RecA in Escherichia coli K-12 using RecA-GFP. Molecular Microbiology. 57, 1074-1085 (2005).

29. A. Kuzminov, F. W. Stahl, Stability of linear DNA in recA mutant Escherichia coli cells reflects ongoing chromosomal DNA degradation. J Bacteriol. 179, 880-888 (1997).

30. Wiktor, J., van der Does, M., Büller, L., Sherratt, D. J. Dekker, C. Direct observation of end resection by RecBCD during double-stranded DNA break repair in vivo. Nucleic Acids Res. 46, 1821-1833 (2018).

31. C. Joo, S. A. McKinney, M. Nakamura, I. Rasnik, S. Myong, T. Ha, Real-Time Observation of RecA Filament Dynamics with Single Monomer Resolution. Cell. 126, 515-527 (2006).

32. J. M. Bork, M. M. Cox, R. B. Inman, RecA Protein Filaments Disassemble in the 5 to 3 Direction on Single-stranded DNA*. Journal of Biological Chemistry. 276, 45740-45743 (2001).

33. Q. Shan, J. M. Bork, B. L. Webb, R. B. Inman, M. M. Cox, RecA protein filaments: enddependent dissociation from ssDNA and stabilization by RecO and RecR proteins. J Mol Biol. 265, 519-540 (1997). 
34. J. Barbé, A. Villaverde, R. Guerrero, Evolution of cellular ATP concentration after UVmediated induction of SOS system in Escherichia coli. Biochem Biophys Res Commun. $117,556-561$ (1983).

35. L. Mohapatra, B. L. Goode, P. Jelenkovic, R. Phillips, J. Kondev, Design Principles of Length Control of Cytoskeletal Structures. Annu Rev Biophys. 45, 85-116 (2016).

36. J. Howard, in Mechanics of Motor Proteins and the Cytoskeleton. Sinauer Associates Inc., U.S. (2001).

37. A. Badrinarayanan, R. Reyes-Lamothe, S. Uphoff, M. C. Leake, D. J. Sherratt, In Vivo Architecture and Action of Bacterial Structural Maintenance of Chromosome Proteins. Science. 338, 528-531 (2012).

38. M.-L. Diebold-Durand, H. Lee, L. B. Ruiz Avila, H. Noh, H.-C. Shin, H. Im, F. P. Bock, F. Bürmann, A. Durand, A. Basfeld, S. Ham, J. Basquin, B.-H. Oh, S. Gruber, Structure of Full-Length SMC and Rearrangements Required for Chromosome Organization. Molecular Cell. 67, 334-347.e5 (2017).

39. L. Wilhelm, F. Bürmann, A. Minnen, H.-C. Shin, C. P. Toseland, B.-H. Oh, S. Gruber, SMC condensin entraps chromosomal DNA by an ATP hydrolysis dependent loading mechanism in Bacillus subtilis. eLife. 4, e06659 (2015).

40. P. Arumugam, S. Gruber, K. Tanaka, C. H. Haering, K. Mechtler, K. Nasmyth, ATP hydrolysis is required for cohesin's association with chromosomes. Curr Biol. 13, 1941-1953 (2003).

41. Wang, X. et al. In Vivo Evidence for ATPase-Dependent DNA Translocation by the Bacillus subtilis SMC Condensin Complex. Mol. Cell 71, 841-847.e5 (2018). 
42. Brandão, H. B., Ren, Z., Karaboja, X., Mirny, L. A. Wang, X. DNA-loop-extruding SMC complexes can traverse one another in vivo. Nat. Struct. Mol. Biol. 1-10 (2021) doi:10.1038/s41594-021-00626-1.

43. A. L. Forget, S. C. Kowalczykowski, Single-molecule imaging of DNA pairing by RecA reveals a three-dimensional homology search. Nature. 482, 423-427 (2012).

44. K. Ragunathan, C. Liu, T. Ha, RecA filament sliding on DNA facilitates homology search. eLife. 1, e00067 (2012).

45. M. R. Evans, S. N. Majumdar, G. Schehr, Stochastic Resetting and Applications. J. Phys. A: Math. Theor. 53, 193001 (2020).

46. J. M. Skerker, M. S. Prasol, B. S. Perchuk, E. G. Biondi, M. T. Laub, Two-Component Signal Transduction Pathways Regulating Growth and Cell Cycle Progression in a Bacterium: A System-Level Analysis. PLoS Biol. 3, e334 (2005).

47. M. Thanbichler, A. A. Iniesta, L. Shapiro, A comprehensive set of plasmids for vanillateand xylose-inducible gene expression in Caulobacter crescentus. Nucleic Acids Research. 35, e137-e137 (2007).

48. B. Ely, Genetics of Caulobacter crescentus. Methods Enzymol. 204, 372-384 (1991).

49. H. Li, R. Durbin, Fast and accurate short read alignment with Burrows-Wheeler transform. Bioinformatics. 25, 1754-1760 (2009).

50. A. R. Quinlan, I. M. Hall, BEDTools: a flexible suite of utilities for comparing genomic features. Bioinformatics. 26, 841-842 (2010). 


\section{Figure Legends}

Fig. 1. Dynamics of RecA filament during homologous recombination. (A) Montage of key events of homologous recombination observed via live-cell imaging of both break/ homology site (MipZ; red) as well as DSB dynamics (RecA; green). Single DSB induction (white asterisk) is marked by the disappearance of one of the two MipZ localizations. RecA nucleation is defined as the period between DSB induction and the initiation of RecA localization at the break site, followed by homology search, marked by the translocation of the RecA filament in the cell. Repair is marked by the loss of RecA filament after search, followed by the reappearance of two MipZ markers. The time line below shows the period of RecA nucleation and loading (after break induction), homology search and repair $(n=100)$. Scale bar: $2 \mu \mathrm{m}$ here and in all other images. (B) Montage of RecA-YFP tracked over time (duration of a single pole-topole traversal event) after induction of a single DSB during homology search (white asterisk). (C) Representative RecA-YFP fluorescence profiles across [top] one pole-to-pole traversal and [bottom] multiple traversals. (D) Centroid position of the RecA filament $\mathrm{x}_{c}$ (relative to cell length) versus time over a single traversal. Plot shows mean and standard deviation from data collected over $n=99$ cells. Mean traversal time $\langle T\rangle=6 \pm 2$ min is indicated. (E) Probability distribution of the centroid steps $\Delta \mathrm{x}_{c}$, the algebraic difference between the centroid positions at two consecutive time points (60 sec apart), shows a bias towards positive values and a weight at $\Delta \mathrm{x}_{c}=0$ (Error bars represent sample error). [inset] Probability distribution of $\mathrm{x}_{c}$ taken over a single traversal cycle $(n=795)$.

Fig. 2. RecN is essential for RecA filament movement in vivo. (A) Montage of RecAYFP tracked over time after induction of a single DSB (white asterisk) - [top] in wild type, [bottom] in cells lacking recN. (B) Representative RecA-YFP fluorescence profiles in a $\Delta r e c N$ background. [top] RecA is tracked for the expected duration of two pole-to-pole traversals; 
[bottom] over expected duration of multiple traversals. (C) Centroid position of the RecA filament $\mathrm{x}_{c}$ versus time for cells lacking recN. Plot shows mean and standard deviation from data collected over $n=50$ cells. (D) Probability distribution of the centroid steps $\Delta \mathrm{x}_{c}$ shows no bias (Error bars represent sample error). [inset] Probability distribution of $\mathbf{x}_{c}$ taken over all time points $(n=852)$, in cells lacking $r e c N$. (E) Representative RecA-YFP fluorescence profile in a $\Delta$ recN background. DSB is induced in non-replicating swarmer cells with a single chromosome, thus lacking a homologous template for repair. (F) Probability distribution of $\mathrm{x}_{c}$ in wild type $(n=911)$ and $\Delta \operatorname{rec} N$ cells $(n=1039)$. DSB is induced in non-replicating swarmer cells with a single chromosome, thus lacking a homologous template for repair.

Fig. 3. RecN-mediated RecA translocation is accompanied by dynamic remodelling of the filament during homology search. (A) Probability distribution of the length changes $\Delta l$, the algebraic difference between the filament lengths at two consecutive time points (60 sec apart) in wild type $(n=347)$ and $\Delta \operatorname{rec} N$ cells $(n=750)$. Error bars represent sample error. (B) Representative images of the types of RecA localizations observed during a wild type traversal, with $(1,2)$ showing puncta and $(4-9)$ showing extended filamentous structures. (C) Joint probability distribution of $l$ and $\mathrm{x}_{c}$ taken over a single traversal ( $n=795$ ). (D) (top) Stochastic kinetic description of RecN-assisted dynamics of RecA filament in terms of its centroid position and length measured in the lab frame. The rates of assembly and disassembly at the two ends of the filament are shown. (bottom) To maintain a finite mean length, the rates of disassembly must exceed the assembly rates at large $l$ (Supplementary Results). Choosing the disassembly rate $k^{--}$to be linear in $l$, and the other rates being constant, allows us to match analytically derived length distribution to the experimentally measured one. (E) The analytically derived distribution $P(l)$ (green) (Supplementary Results) is compared with the experimental data for wild type (plotted as a density histogram) (fit parameters presented in the Supplementary Results). [inset] The theory predicts a relation between the centroid velocity $v_{c}$ and mean filament length $\langle l\rangle$. 
Dashed red line highlights the relationship between $v_{c}$ and $\langle l\rangle$, estimated Pearson correlation coefficient $r=0.236$ (p-value $=0.045,95 \%$ CI $[0.00597689,0.441833]$ )(for this estimate we have excluded outliers).

Fig. 4. RecN ATPase cycle regulates RecA filament dynamics. (A) Montage of RecAYFP tracked over time after induction of a single DSB (white asterisk) for RecN ATP hydrolysis mutant $\left(r e c N^{E 473 Q}\right)$. (B) Representative montage of RecA-YFP tracked over time after induction of a single DSB (white asterisk) for RecN ATP binding mutant $\left(r e c N^{D 472 A}\right)$. (C) Physical interaction between RecN mutants and RecA is confirmed using the bacterial-twohybrid assay. Representative images from three independent repeats. Images for RecA-RecN and empty vector interactions are from Fig. S3D. (D) Sensitivity of $r e c N^{D 472 A}$ and $r e c N^{E 473 Q}$ to DSB-inducing agent norfloxacin is evaluated (in comparison to wild type, $\Delta r e c N$ and $\Delta r e c A$ cells, from Fig. S3E). Representative image from at least three independent repeats. (E) [top] Representative RecA-YFP fluorescence profile across the length of the cell in a $r e c N^{E 473 Q}$ background. [bottom] Representative RecA-YFP fluorescence profile across the length of the cell in a recN $N^{D 472 A}$ background. (F) Position of centroid of RecA filament normalized to length of the cell $\left(\mathrm{x}_{c}\right)$ is plotted for $\operatorname{rec} N^{E 473 Q}$. Mean and standard deviation is shown $(n=50)$. (G) As (F) for $r e c N^{D 472 A}$. (H) Density histogram of RecA filament lengths $(l)$ across all time points of imaging for wild type, $r e c N^{D 472 A}$, and $r e c N^{E 473 Q}(n=229)$. Distribution of $l$ was found to be significantly different between wild type and $\operatorname{rec}^{E 473 Q}$ (p-value $=0.0001,95 \%$ CI [-0.004229, 0.06469], Welch's t-test). Distribution of $l$ was comparable between wild type and $r e c N^{D 472 A}$ $(\mathrm{p}$-value $=0.1535,95 \%$ CI $[-0.003326,0.02108])$.

\section{Supplementary content}

Supplementary Results with detailed description of theory and quantitative analysis.

Supplementary figures (associated with main text). 
bioRxiv preprint doi: https://doi.org/10.1101/2021.08.16.456443; this version posted August 16, 2021. The copyright holder for this preprint (which was not certified by peer review) is the author/funder, who has granted bioRxiv a license to display the preprint in perpetuity. It is made available under aCC-BY-NC-ND 4.0 International license.

Supplementary videos 1-6.

Supplementary tables $1-3$. 


\section{Figure 1}

A

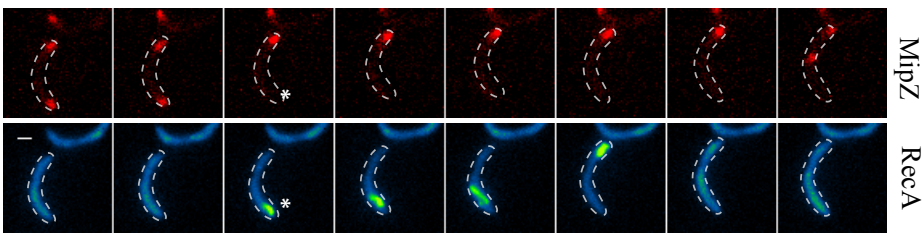

DSB induction + end resection

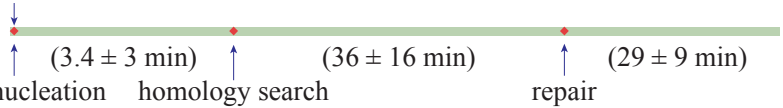

RecA nucleation homology search repair

B

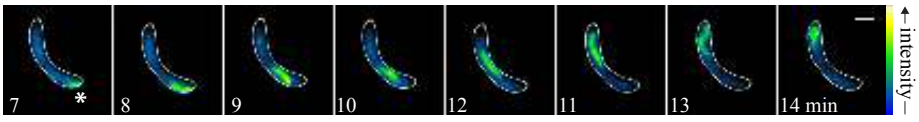

RecA (homology search)

C

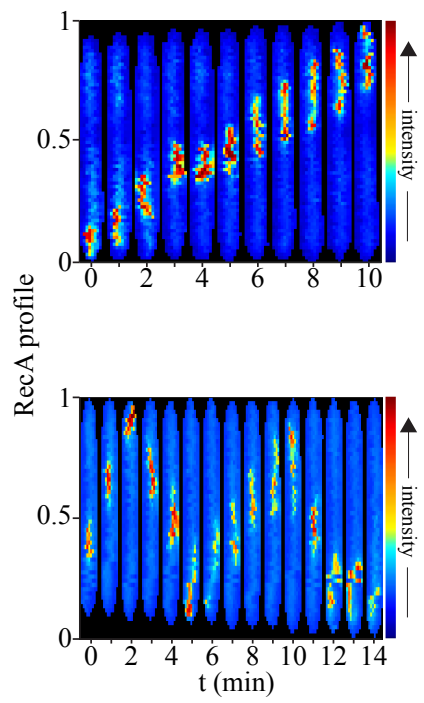

D

$\mathrm{x}_{\mathrm{c}}=$ position of filament centroid/ $\mathrm{L}$

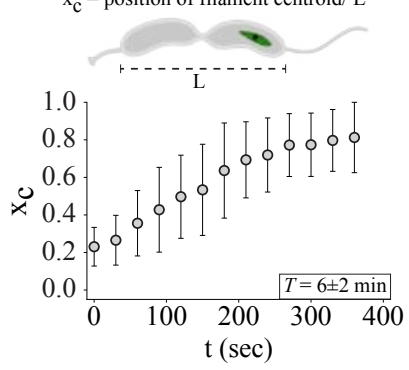

$\mathbf{E}$

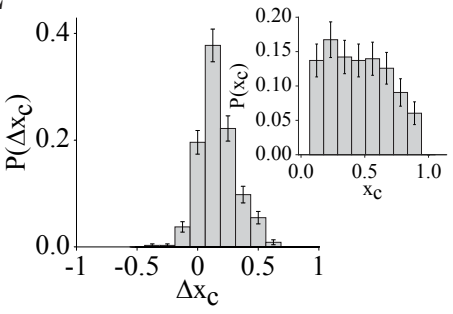


A

Figure 2
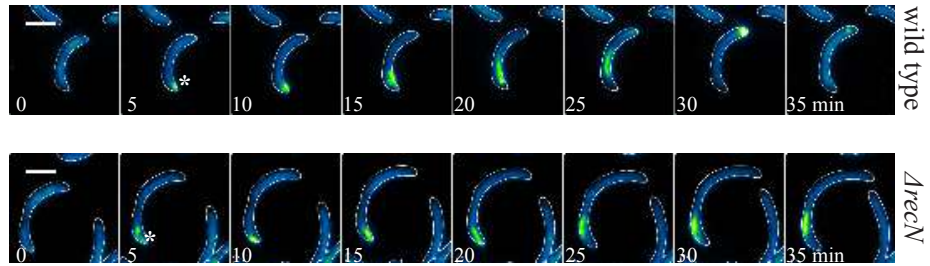

B

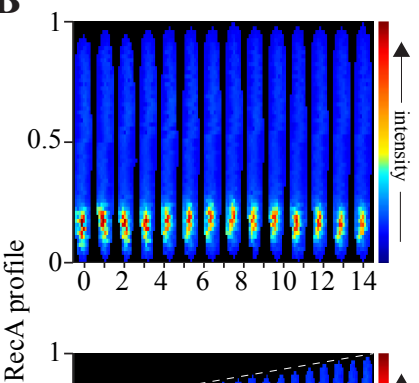

0.5

0

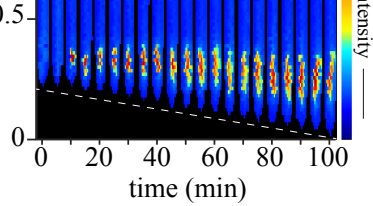

E

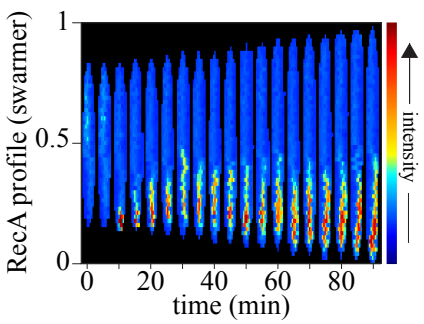

C

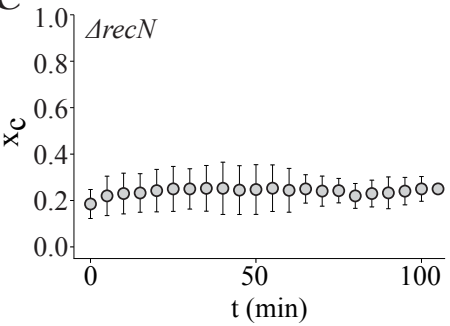

D

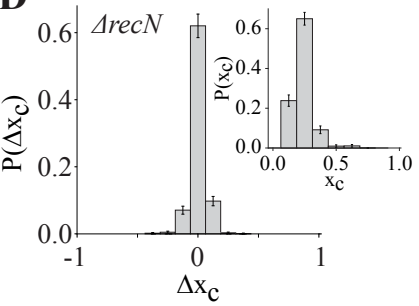

F

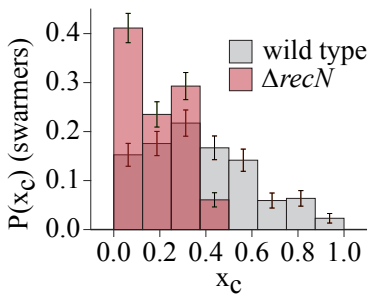


Figure 3

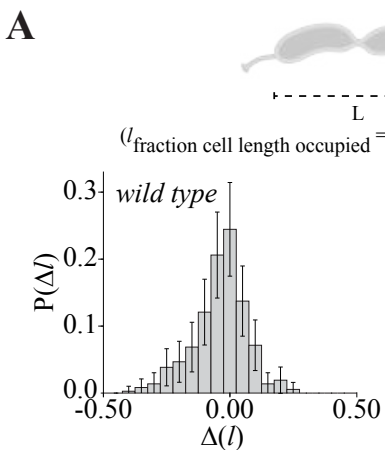

C

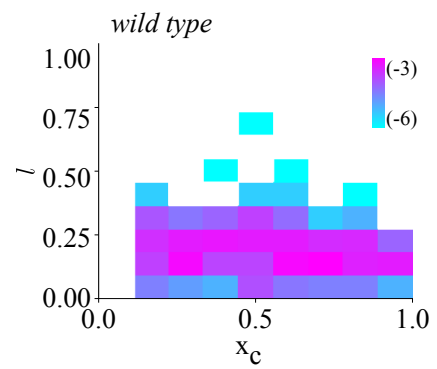

B

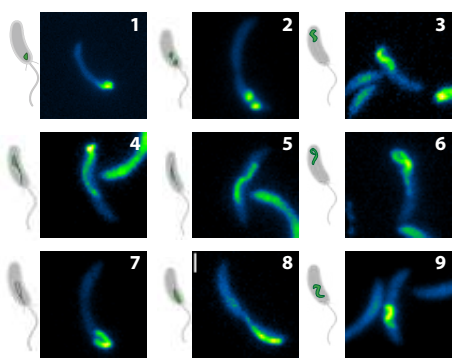

RecA localizations (homology search)

E
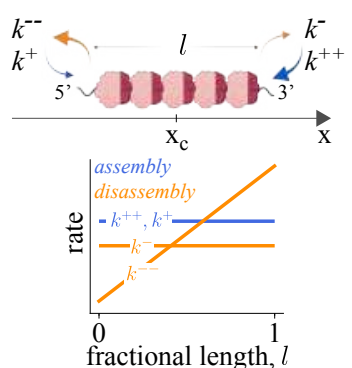

wild type

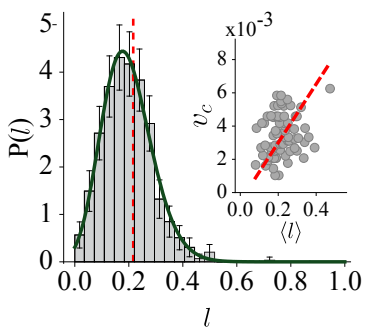


Figure 4

A

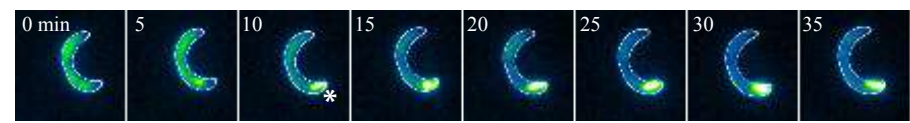

$\operatorname{recN}^{E 473 Q}$

C

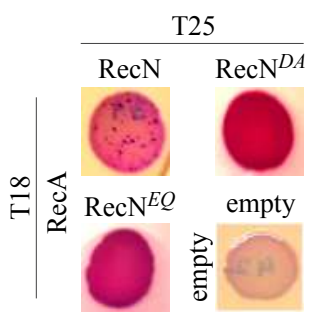

D
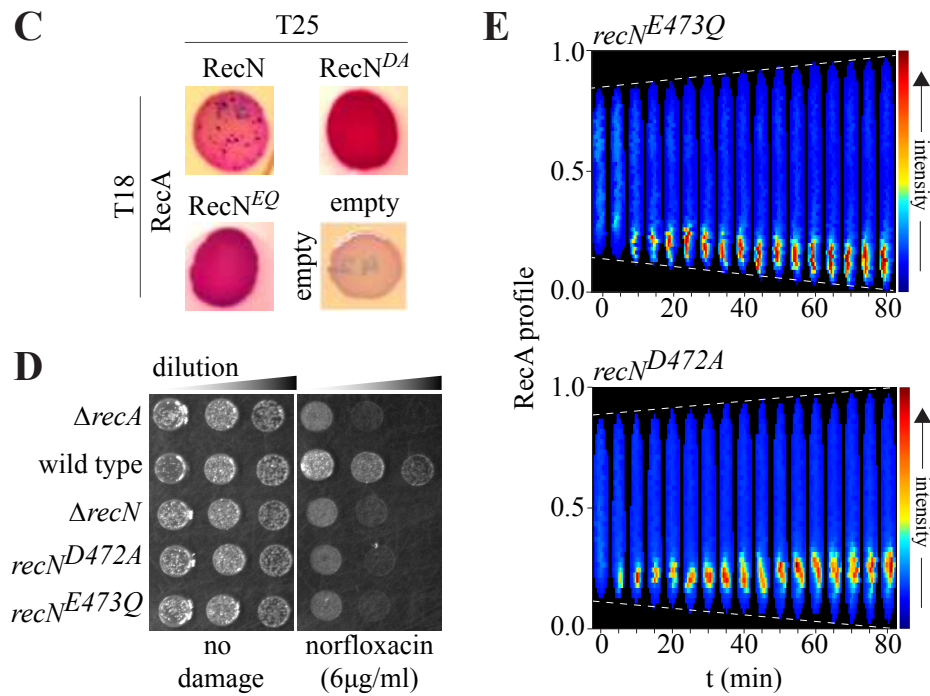

B

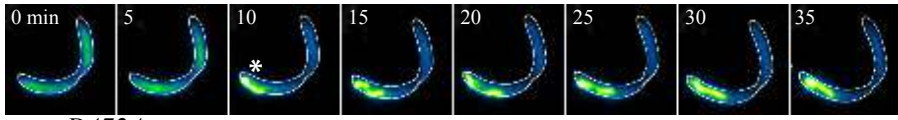

$\operatorname{rec}^{D 472 A}$

F
G
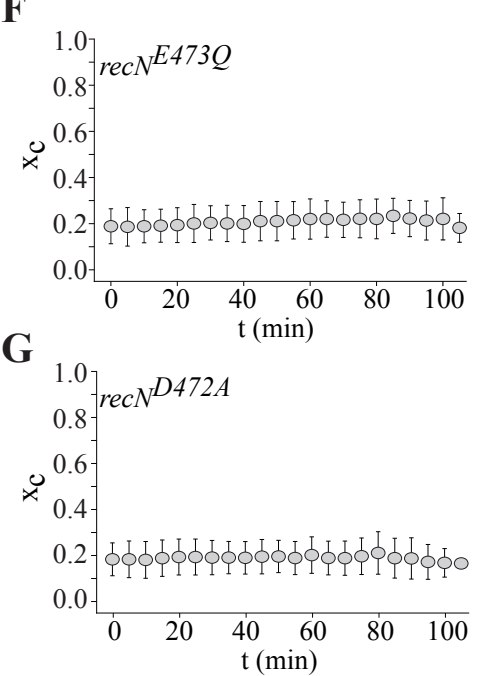

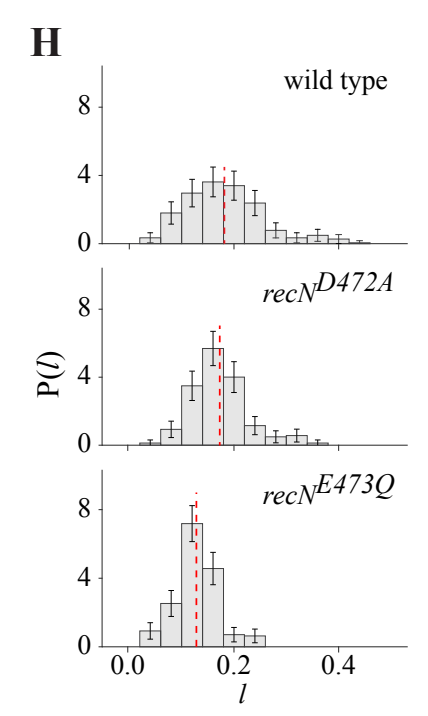

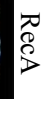

